\title{
Effect of wall on subsonic non-circular jets
}

\author{
${ }^{1}$ Bhatia Devansh Pradeep, ${ }^{2}$ Imanbir Singh, ${ }^{3}$ Sonam Eden Bhutia
}

\author{
${ }^{1,2,3}$ B.Tech Student, Dept. of Aerospace Engineering, SRM Institute of Science and Technology, Chennai,
} Tamil Nadu, India

\begin{abstract}
The present experimental study aims at characterizing the mixing characteristics of subsonic elliptic and rectangular orifice jets $\left(\mathrm{V}_{\mathrm{e}}=51 \mathrm{~m} / \mathrm{s}\right)$ in the presence of wall of different surface roughness, namely smooth, fine and coarse, placed parallel to the jet axis at the edge of the orifice exit. The wall of length $120 \mathrm{~cm}$ of different surface roughness was oriented either parallel to the minor axis plane or major axis plane if the orifice. The wall had a strong effect on the mixing characteristics of elliptic and rectangular jets. The centerline velocity measurement showed that the potential core of elliptic and rectangular orifice jets was around $3 \mathrm{D}_{\mathrm{e}}$ and $3.5 \mathrm{D}_{\mathrm{e}}$. For both jets, all the wall configurations retarded the mixing of jet with the ambient fluid only in the characteristic decay zone but not in the core and fully developed zones. The level of mixing retardation was found to be maximum when wall was placed parallel to the major axis plane of the jet. The effect of wall on jet mixing was intense for rectangular jet compared to elliptic jet.
\end{abstract}

Keywords: Subsonic, Surface roughness, Wall configuration, Elliptical jets, Rectangular jets.

\section{Introduction}

\subsection{Overview}

Flow jets are flow issued from a small opening at a high velocity and represents an imperative area of research interest. The phenomenon covers wide range of practical and engineering applications including space propulsion, boundary layer separation control, and heat transfer as a method of achieving particularly high heat transfer coefficients, in drying processes, air curtains for room conditioning, heating and ventilating applications. Flow jets are broadly studied under three categories, viz., free jet, wall jet, and impinging jet. This classification is based on presence of a solid surface (i.e. wall) against the jet expelled. In free jet configuration, the jet is issued into an unbounded fluid (stationary or moving). The impinging jet refers to jet striking a plane solid surface in its path at any given orientation. The phenomenon finds application in problems as the mixing and combustion of atomized liquid fuel, jet cooling of commercial products, and the problems associated with jets issuing from rockets or vertical take-off and landing aircraft. Various types of jet flows injected into the turbulent boundary layer are applied as a method for boundary layer control and interactions between jets and boundary layers are also of interest as complex turbulent flows. The jets are usually injected into the boundary layer either perpendicular to the wall or parallel to it. The flows treated in this study are parallel to the wall.

Studies on two-dimensional mixing of jet with a wall flow have been performed, some in relation to film cooling of gas turbine blades, and others involving the separation prevention in diffuser. However, the two-dimensional injection is difficult in terms slit construction, and if the three dimensional mixing utilizing a circular pipe is effective, it may be more practical than the two dimensional injection. In the present study we have investigate experimentally the effect of wall on non-circular orifices (elliptical and rectangular) when the wall is laced parallel to the flow. The experiments were carried out on both smooth and rough wall. We observed axis switching while carrying out the experiments.

\subsection{Free JET}

Free jets can be defined as a pressure driven unrestricted flow of a fluid into a quiescent ambience. As the fluid moves downstream, it interacts with the surrounding fluid and 
momentum drops. Subsequently, the jet efflux splits into three regions viz., core, entrainment, termination, all related to centerline velocity decay.

\subsection{Wall JET}

A plane wall jet is obtained by injecting fluid parallel to a wall in such a way that the velocity of the fluid, over some distance from the wall, supersedes that of the ambient flow. The structure of a turbulent wall jet can be described as being composed of two canonical shear layers of different types. The inner shear layer reaching from the wall out to the point of maximum stream wise velocity, resembles a boundary layer, while the outer layer, from the maximum velocity out to the ambient fluid, resembles a free shear layer. A consequence of the double shear layer structure is that properties such as momentum transfer and mixing will exhibit distinctively different characteristics and scaling properties in the two shear layers.

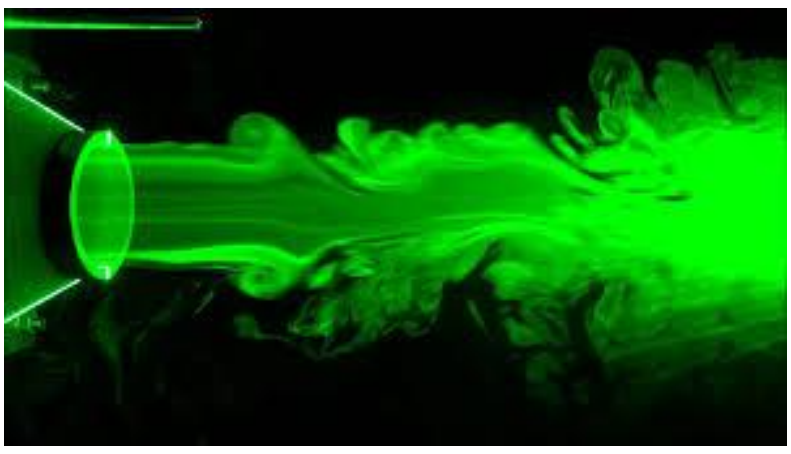

Fig. 1.3.1 Pictorial view of flow jet

\subsection{Regions of JET}

In potential core region, the centerline velocity is almost equal to the exit or outlet velocity. This region normally extends up to $4 \mathrm{~d}$ to $6 \mathrm{~d}$, where $\mathrm{d}$ is the diameter of the nozzle exit. In entrainment region, fluid comprehensively interacts with the surrounding fluid and is identified by drastic change in flow characteristics. Shear layer is the region in which most of the interactions and mixing between the ambient and jet fluids take place. The entrainment region comprises of two distinct zones viz., transition and self-similar. In transition zone, the centerline velocity starts to decay. The velocity decay can be approximated as proportional to $\mathrm{x}-0.5$, where $\mathrm{x}$ is the axial distance. This usually corresponds to a region from $6 \mathrm{~d}$ to $20 \mathrm{~d}$, and it is known as the interaction region where shear layers from both sides merge. In the self-similar zone, the transverse velocity profiles are similar at different values of $\mathrm{x}$ and the centerline velocity decay is approximately proportional to $\mathrm{x}-1$. Final region is the flow termination where, the centerline velocity decays rapidly to stagnation. Although this zone has been studied by several researchers, the actual mechanisms in this zone are not understood properly. It is important to note that, with distance, the efflux loses momentum and the length of core region varies under different conditions. However, the flow characteristics of jet efflux while impinging on a solid surface are very likely to alter than the free jet. The surface when placed at different locations and orientations will have significant implications. Thus, impinging jets have attracted much research from the viewpoint of the fluid flow characteristics and their influence on energy transfer. An impinging flow jet is similar to free jet and can be identified in three zones viz., free jet, stagnation, wall jet. Free jet zone represents region that is largely unaffected by the presence of the impingement surface. A potential core exists within which the jet exit velocity is conserved. A shear layer exists between the potential core and the ambient fluid which entrains ambient fluid and causes the jet to spread radially resulting lower mean velocity than the jet exit velocity. Beyond the potential core the shear layer has spread to the point where it has penetrated to the centerline of the jet. At this stage, the centerline velocity decreases drastically. Second is the stagnation zone that extends to a radial location defined by the spread of the jet. The stagnation zone includes the stagnation point where the mean velocity is zero and within this zone the free jet is deflected into the wall jet flow. Finally, the wall jet zone which extends beyond the radial limits of the stagnation zone.

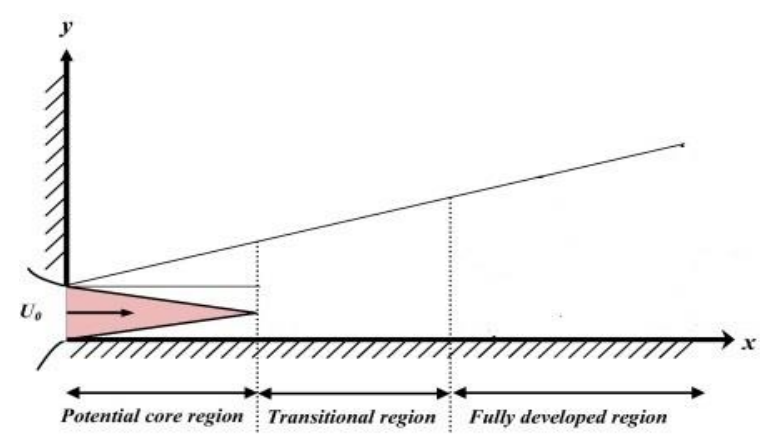

Fig. 1.4.1 Regions of jet

\section{Literature Survey}

1. Ezhil Manickam, Pratik Tiwari, Shitiz Sehgal, Ajay Babusekar , Vinayak Malhotra, "An experimental analysis on the coupling effect of center wall location and side walls orientation on impinging flow jets." International Journal of Application or Innovation in Engineering \& Management, Volume 6, Issue 6, June 2017 
The work aims at understanding the effect of coupling of central wall location and side walls orientation on flow characteristics of an impinging flow jet. The study experimentally intends physical insight into heterogeneous phenomena of efflux from a small opening impinging on a surface confined from three sides under deviant conditions. Systematic experiments were performed on a cascade tunnel with flow ejected at a velocity of $36 \mathrm{~m} / \mathrm{s}$. Results show that outside the core region, the flow experiences a monotonic reduction with increase in distance along streamline and radial direction. The flow features vary distinctly with three wall configurations than conventional wall jet. The center wall location is more efficient in bringing substantial change when placed closer to the exit (low velocity losses) and primarily governs the chances of strong flow deflection or back flow losses. However, wall placed far away from exit results in diminishing returns with a critical value beyond which the flow characteristics become insensitive. The presence of walls on sides results in reduction in the core region. The geometry was noted to influence the entrainment of ambient fluid, the spread of the shear layer and the length of the potential core.

2. Glauret, M. B., "The wall jet," Journal of Fluid Mechanics, vol. 1, pp. 625-643, 1956

This paper considers the flow due to a jet spreading out over a plane surface, either radially or in two dimensions. Solutions of the boundary layer equations are sought, according to which the form of the velocity distribution across the jet does not vary along its length. For laminar flow, such a similarity solution is obtained explicitly. For turbulent flow, an eddy viscosity is introduced, and it is eventually seen that complete similarity is not attainable, but that confident predictions can nevertheless be made about the nature of the velocity distribution and the rate of growth of the wall jet.

\section{Methodolgy}

A simple apparatus was adapted and modified for present study. The apparatus consisted of a) jet tunnel and Pitot tube b) Digital manometer. The jet tunnel issues a free air jet using a centrifugal blower with a velocity of $\sim 36 \mathrm{~m} / \mathrm{s}$. The efflux is from a small rectangular opening $(192 \mathrm{~mm} \mathrm{x}$ $105 \mathrm{~mm}$ ) into the air in a quiescent room. The jet issuing from the tunnel was made to of equal area for both the non-circular jets (i.e. elliptical and rectangular). To calculate the area, the circular shape area was calculated and equated with the non-circular shapes. The calculation is shown below,

To calculate the diameter of circle:

Exit area of jet tunnel $>$ area of circle

$192 * 105>\pi r^{2}$

$\mathrm{r}=40 \mathrm{~mm}$

$D_{\mathrm{e}}=80 \mathrm{~mm}$

To compare non circular shapes efficiently, we keep area constant

Elliptical area:

Area of circle $=$ Area of ellipse

$\pi \mathrm{r}^{2}=\pi \mathrm{ab} \quad$ (where, $\mathrm{a}=$ semi-major $; \mathrm{b}=$ semi-minor axis)

We take the aspect ratio as 2 (i.e. $a=2 b$ )

$\mathrm{r}^{2}=\mathrm{ab}$

We get, $\mathbf{a}=\mathbf{5 6 . 5 6 8} \mathrm{mm} ; \quad b=\mathbf{2 8 . 2 8} \mathrm{mm}$

Now to get the area of the rectangle

Area of the rectangle $=$ Area of ellipse

Length*Breadth $\quad=50.285 \mathrm{~cm}$

Taking, Aspect ratio as 2:1

$2 \mathrm{~B}^{2}=50.285 \mathrm{~cm}$

Therefore,

$B=50.133 m m$

Thus,

\section{$\mathrm{L}=100.265 \mathrm{~mm}$}

For wall,

To design the wall, we considered the near field of the flow which was till $15 \mathrm{D}_{\mathrm{e}}$ and at $15 \mathrm{D}_{\mathrm{e}}$ the spread of the flow was maximum $35 \mathrm{~cm}$ without the wall for elliptical and rectangular so the breadth of the wall was fixed at $40 \mathrm{~cm}$.

The material of the wall is glass as the surface of the glass is smooth.

Length of the wall $=120 \mathrm{~cm}$

Breadth of the wall $=40 \mathrm{~cm}$ 


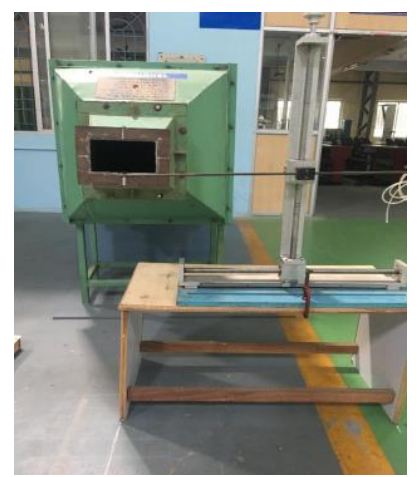

Fig 3.1 Jet Tunnel

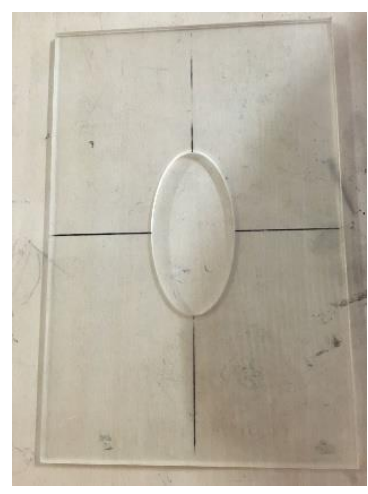

Fig 3.2 Elliptical Orifice Orifice

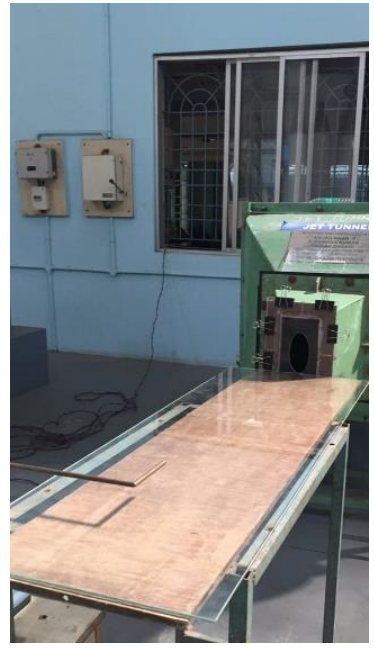

Fig 3.4 Smooth wall (parallel to minor axis of elliptical orifice)

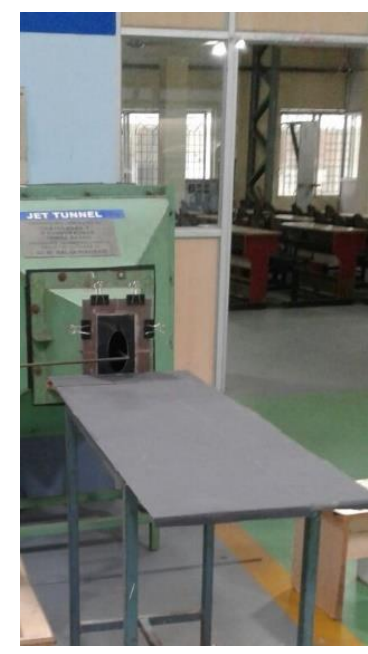

Fig 3.5 Fine surface wall (parallel to minor axis of elliptical orifice)

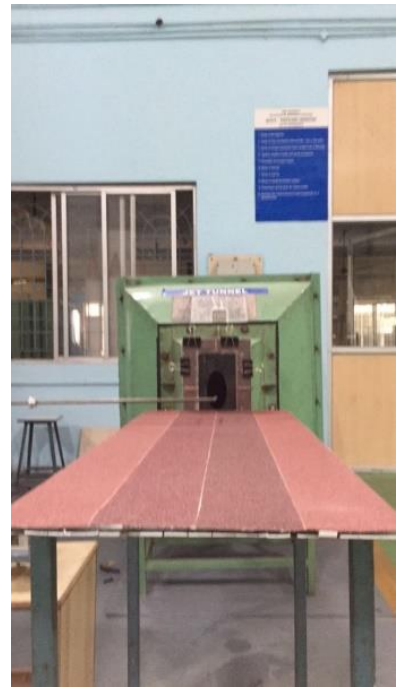

Fig 3.6 Rough surface wall (parallel to minor axis of elliptical orifice)

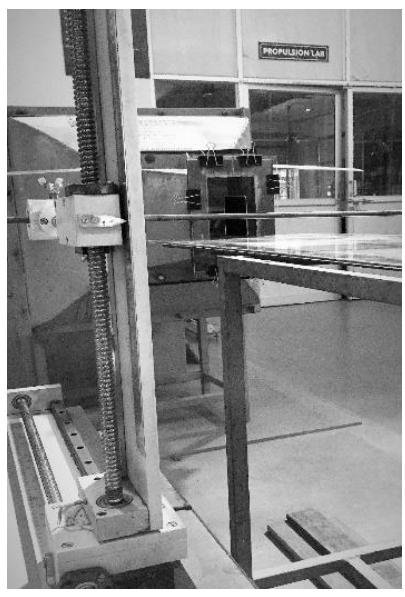

Fig 3.7 Smooth wall (parallel to minor axis of rectangular orifice) 


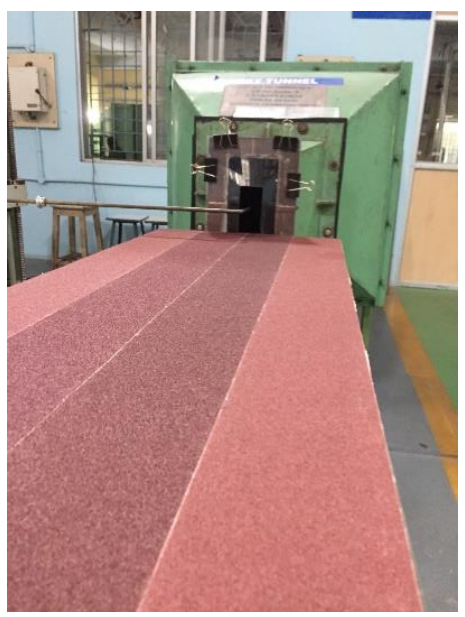

Fig 3.8 Rough wall (parallel to minor axis of rectangular orifice)

\section{RESULTS AND DISCUSSION}

\subsection{Centerline graphs for elliptical orifice $(X-$ axis)}

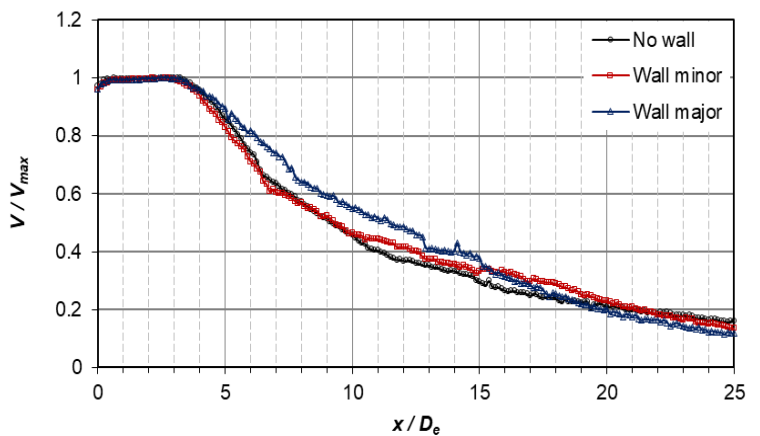

Fig. 4.1.1 Elliptical Centerline Velocity for Smooth Wall

The graph in Fig.5.1.1 represents the comparison between with and without wall when the surface of the wall is smooth and the orifice is elliptical. It can be seen that when the smooth wall is placed parallel to major and minor axis the mixing characteristic of the elliptical jet is modified, but it has only been modified in the characteristic decay region. The core and fully developed region remains the same. The core length with or without wall remains to be $3 D_{e}$ i.e. $24 \mathrm{~cm}$ away from the exit of the orifice. It can be seen that when the wall is paced parallel to the flow the mixing retards in the characteristic decay region as the value of the $\mathrm{V} / \mathrm{V}_{\max }$ ration increases. The retardation is seen to be more intense when the wall is placed parallel to the major axis. When wall is placed parallel to the minor axis it can be seen that the retardation occurs only after $10 \mathrm{D}_{\mathrm{e}}$ i.e. $80 \mathrm{Cm}$ from the exit of the orifice. Up till then it shows same characteristic as shown by the case where no wall is placed. No wall certainly helps to enhance the mixing.

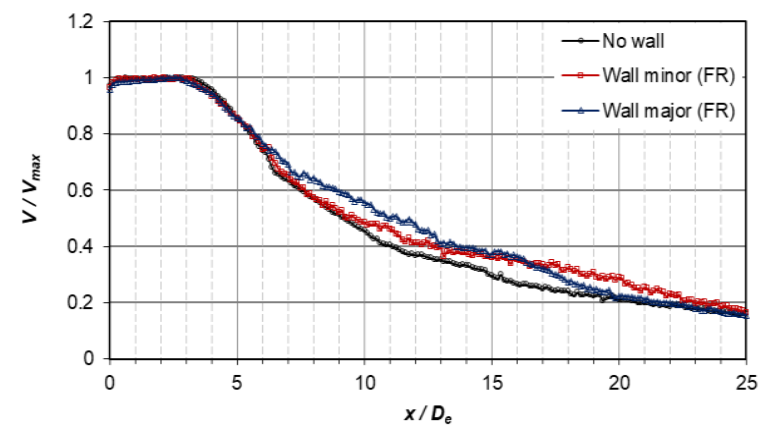

Fig. 4.1.2 Elliptical Centerline Velocity for Fine Emery Wall

The graph in Fig. 4.2 shows the centerline velocity profile comparison for elliptical jets with and without wall. The surface of the wall is covered with fine emery sheet to make the surface a little rough. Like the smooth wall it can be observed that the core and fully developed region remain the same while the mixing gets affected only in the characteristic decay region. Here we can see that when the wall is placed parallel to the minor axis it is behaving same as the no wall case but only till $9 \mathrm{D}_{\mathrm{e}}$ after which it also starts to retard the mixing. It is interesting to note that after $16 \mathrm{D}_{\mathrm{e}}$ the mixing is more retarded when the wall is placed parallel to the minor axis, which can also be noted in smooth wall case.

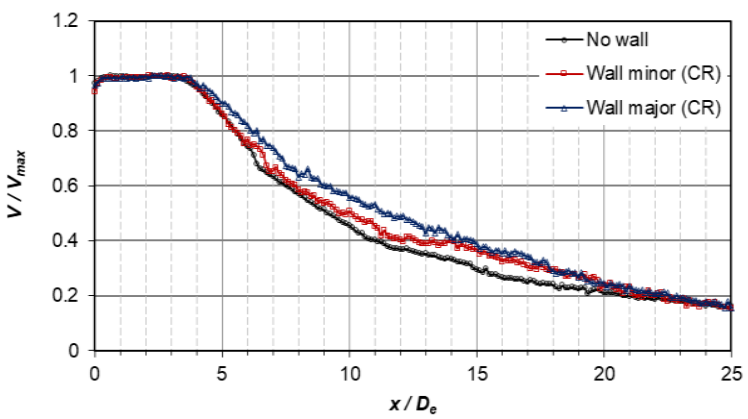

Fig. 4.1.3 Elliptical Centerline Velocity for Coarse Emery Wall

The graph in Fig. 4.3 represents the comparison of elliptical jet in the presence of wall and without wall when the surface of the wall is covered with coarse emery sheet. Here also it can be noticed that the core and fully developed region are the same, and it is the characteristic decay region which helps us to evaluate results. It can be clearly seen that the retardation is more intense when the wall is placed parallel to the major axis. When wall is placed parallel to the minor axis it shows the same characteristics as the no wall case but only till $7 \mathrm{D}_{\mathrm{e}}$, after that it also start to retard the 
flow. In contrast to the earlier cases it can be noted that the curves for major and minor axis overlap with each other after $17 \mathrm{D}_{\mathrm{e}}$. Then after 20 $D_{\mathrm{e}}$ all the three curves start to overlap.

\subsection{Centerline graphs for rectangular orifice (X-axis)}

For rectangular orifice the trends are sought of same. The differences are, it can be noted that the when the wall is placed parallel to the minor axis it shows the same trend that of no wall but only till $7 \mathrm{D}_{\mathrm{e}}$, after which it starts to retard. The retardation is seen to be more intense when the wall is placed parallel to the major axis as it starts to retard right after the core region which is not seen in elliptical jets. In elliptical jets when wall is placed parallel to the major axis it starts to retard after $5 \mathrm{D}_{\mathrm{e}}$. The retardation is seen to be more in rectangular case as it has higher values of $\mathrm{V} / \mathrm{V}_{\max }$ ratio.

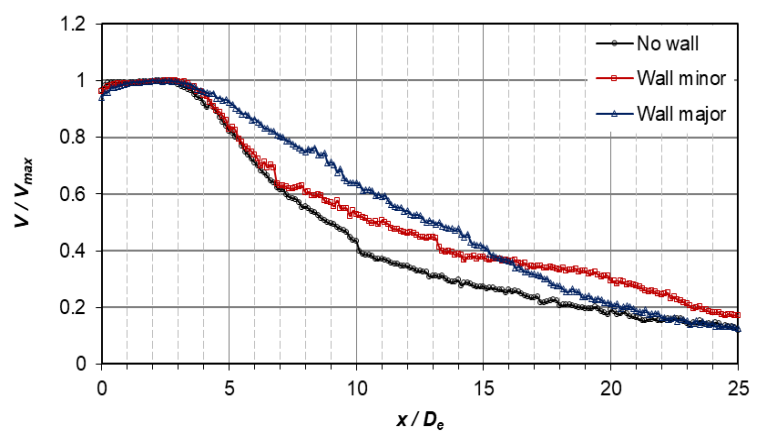

Fig.4.2.1 Rectangular Centerline Velocity for Smooth Wall

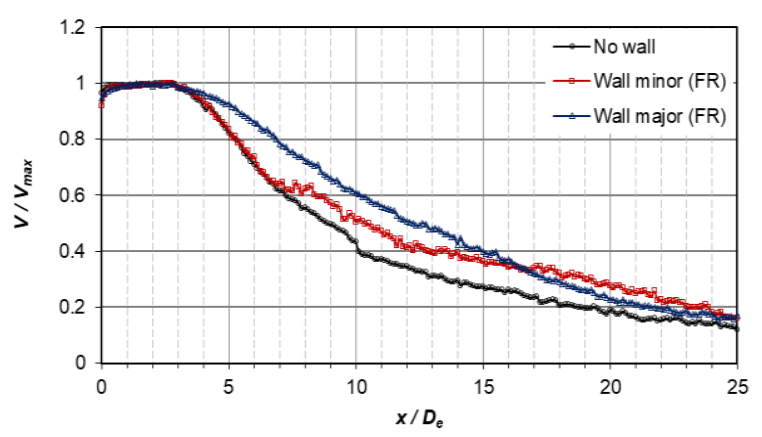

Fig. 4.2.2 Rectangular Centerline Velocity for Fine Emery Wall

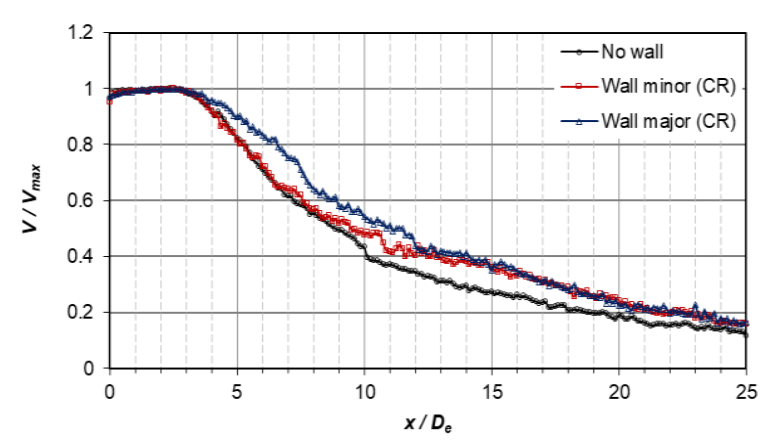

Fig. 4.2.3 Rectangular Centerline Velocity for Coarse Emery Wall

\subsection{Centerline graphs for wall placed parallel to major axis of elliptical and rectangular orifice (X-axis)}

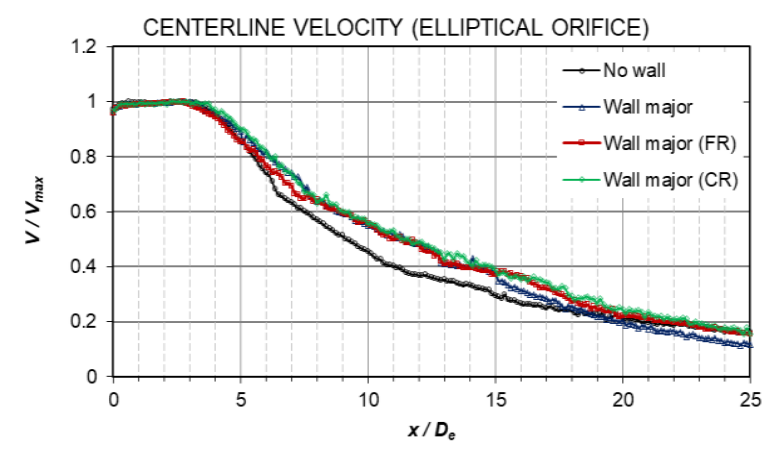

Fig. 4.3.1 Elliptical Centerline Velocity When wall is placed parallel to major axis

The graph in Fig 4.3.1 shows the comparison between the centerline curves when the wall is placed parallel to the major axis when we vary the surface roughness of the wall with No wall. The three surface roughness cases chosen here are smooth, fine and coarse. As discussed earlier the core and the fully developed region remains the same. The mixing gets affected only in the characteristic decay region. Here if we see Fine emery follows the trend of no wall but it starts to retard after $6 \mathrm{D}_{\mathrm{e}}$, while the coarse emery sheets starts to affect right at the starting of characteristic decay. Both fine and coarse emery curves overlap after $8 \mathrm{D}_{\mathrm{e}}$ and they continue to overlap until end. It is very interesting to note that after $15 \mathrm{D}_{\mathrm{e}}$ smooth wall curve starts to show less $\mathrm{V} / \mathrm{V}_{\max }$ ratio which represents that after $15 \mathrm{D}_{\mathrm{e}}$ smooth wall increases the mixing and after $20 \mathrm{D}_{\mathrm{e}}$ it is showing good mixing characteristics than the no wall case. 


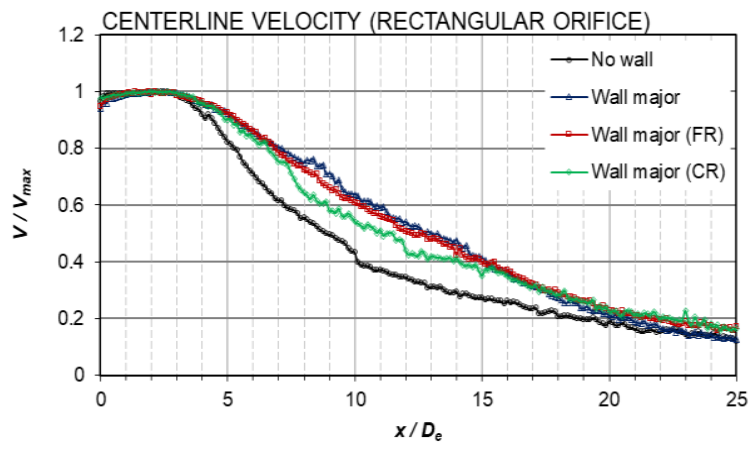

Fig. 4.3.2 Rectangular Centerline Velocity When wall is placed parallel to major axis

The graph in Fig. 4.3.2 shows the centerline velocity profile for rectangular case when wall is placed parallel to the major axis while varying the surface roughness. It is compared with the no wall case. Here it can be noted that retardation is more as compared to elliptical jets. It is also contrary to see that the coarse emery is affecting less than the fine and smooth wall. All the curves merge after $20 \mathrm{D}_{\mathrm{e}}$. It can also be noticed that smooth wall and fine wall curve remain overlapped with each other from starting and have retarded the flow more

\subsection{Centerline graphs for wall placed parallel to minor axis of elliptical and rectangular orifice (X-axis)}

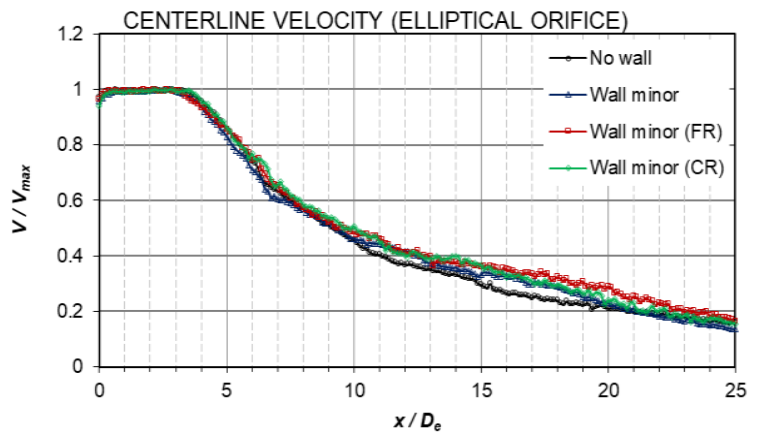

Fig. 4.4.1 Elliptical Centerline Velocity When wall is placed parallel to minor axis

The graph in Fig. 4.4.1 shows the centerline velocity plot for elliptical jets when it is placed parallel to the minor axis while varying the surface roughness. It is compared with no wall. As we can see that the mixing is not getting much affected when wall is placed parallel to the minor axis. All regions are the same ad all curves are overlapping with each other. The only change which can be noticed is between 10 and $20 \mathrm{D}_{\mathrm{e}}$ which is part of characteristic decay. The wall starts to retard the mixing after $10 \mathrm{D}_{\mathrm{e}}$. Smooth and coarse wall curve overlap with each other. It is interesting to notice that after $15 \mathrm{D}_{\mathrm{e}}$ the fine wall retards the mixing more which is contrary to major axis case.

CENTERLINE VELOCITY (RECTANGULAR ORIFICE)

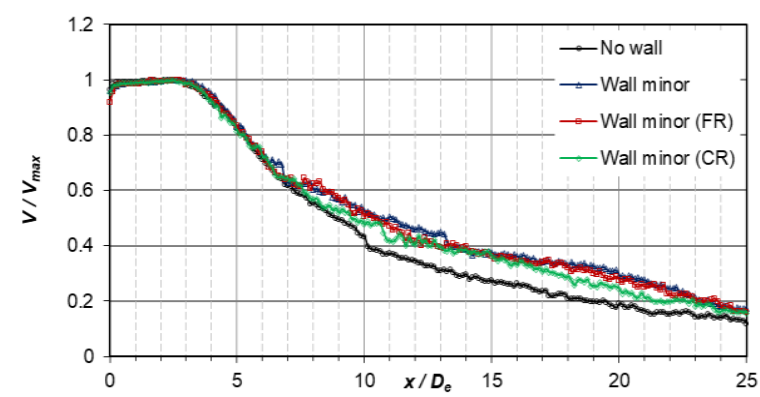

Fig. 4.4.2 Rectangular Centerline Velocity When wall is placed parallel to minor axis

The graph in Fig. 4.4.2 shows the centerline velocity profile for rectangular case when wall is placed parallel to the minor axis while varying the surface roughness. It is compared with the no wall case. As in the case of wall placed parallel to the major axis, here also the coarse sheet is affecting less as compared to fine and smooth wall. Fine and smooth wall stay overlapped with each other and after $17 \mathrm{D}_{\mathrm{e}}$ they start to retard the flow more. It is also very interesting to see that all the curves overlap with each other after $23 \mathrm{D}_{\mathrm{e}}$ which is contrary to all other cases where all the curves start to overlap after $20 \mathrm{D}_{\mathrm{e}}$. The retardation is surely more as compared to the elliptical case.

\subsection{Axial Switching Graphs}

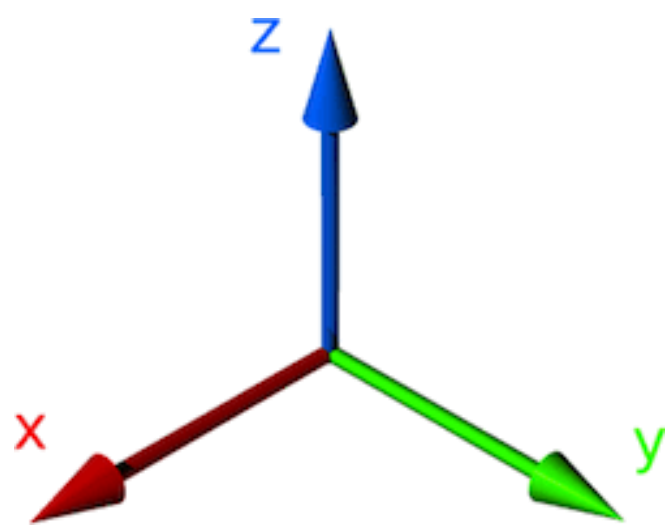

$\mathrm{Y}$ and $\mathrm{Z}$ axis plots helps us to recognize positions where axial change occurs. These graphs were plotted till $25 \mathrm{D}_{\mathrm{e}}$.

The axial change occurs between $1 \mathrm{D}_{\mathrm{e}}$ and $3 \mathrm{D}_{\mathrm{e}}$ for almost all cases but I some cases it occurs between $3 \mathrm{D}_{\mathrm{e}}$ and $5 \mathrm{D}_{\mathrm{e}}$. 
It is noted as when the wall is placed parallel to major axis the number of readings should be more in $\mathrm{Z}$ direction and when placed parallel to minor axis, $\mathrm{Z}$ direction should have more readings. Axial change would mean that more number of readings are coming in the opposite direction.

For Smooth wall, when it is placed parallel to major axis the axial change occurs between $0 \mathrm{D}_{\mathrm{e}}$ and $1 \mathrm{D}_{\mathrm{e}}$ as more number of readings are coming in $\mathrm{Y}$ direction which is opposite as more number of readings should come in $\mathrm{Z}$ direction.

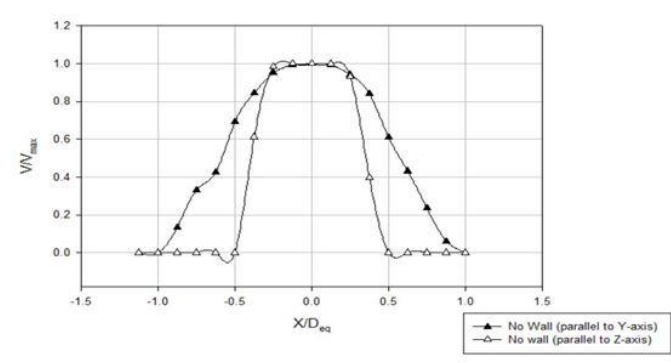

Fig 4.5.1. Axial switch graph for smooth wall (elliptical) parallel to major axis.

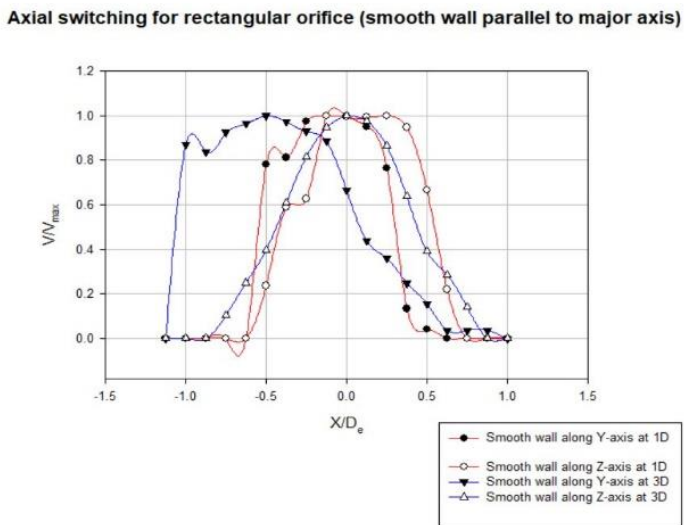

Fig. 4.5.2. Axial switching graph for smooth wall (rectangular) parallel to major axis.
Axial switching for elliptical orifice (smooth wall parallel to minor axis)

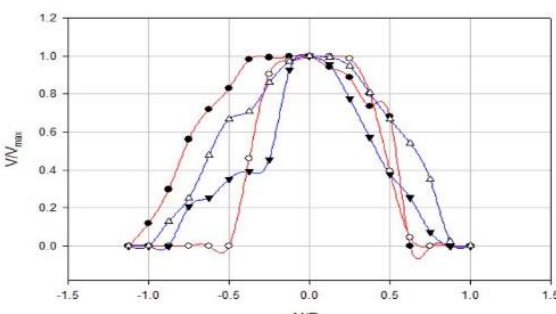

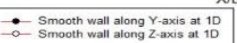

- Smooth wall along $Y$-axis at 30
$\therefore$ Smooth wall along $Z$-axis at 30

Fig 4.5.3. Axial switching graph for smooth wall (elliptical) parallel to minor axis.

Axial switching for rectangular orifice (smooth wall parallel to minor axis)

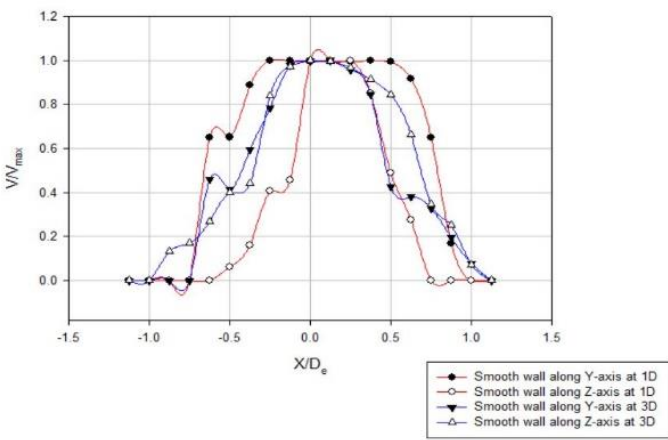

Fig 4.5.4. Axial switching graph for smooth wall (rectangular) parallel to minor axis.

Axial switching for elliptical orifice (Fine wall parallel to minor axis)

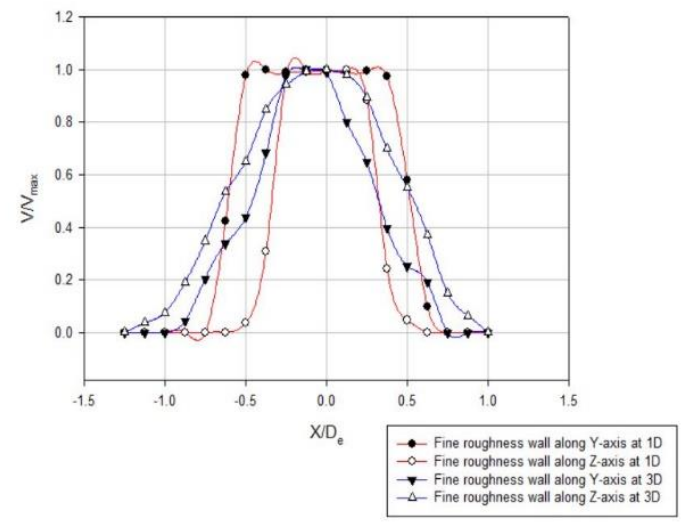

Fig 4.5.5. Axial switching graph for Fine wall (elliptical) parallel to minor axis. 


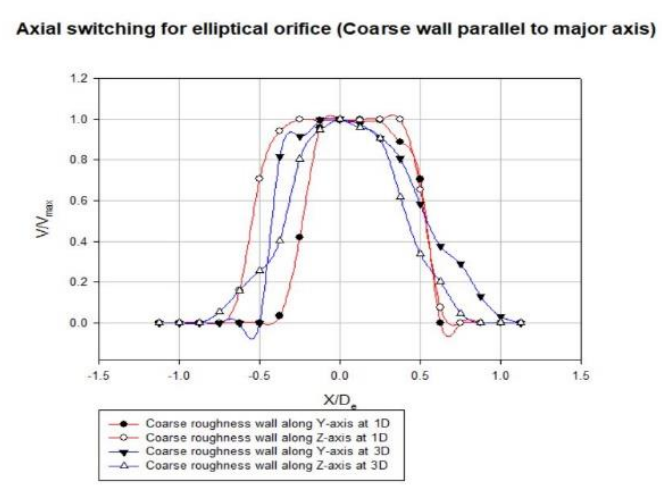

Fig 4.5.6. Axial switching graph for Rough wall (elliptical) parallel to major axis.

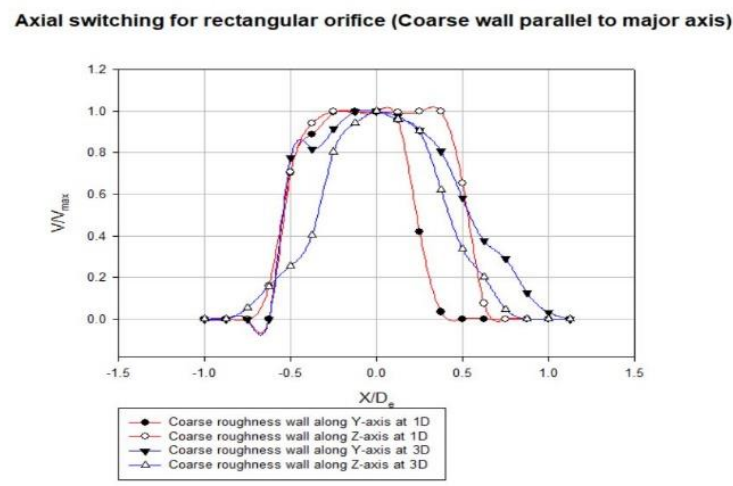

Fig 5.3.7 Axial switching graph for Rough wall (Rectangular) parallel to major axis.

\section{Conclusion}

1. For both jets, all the wall configurations retarded the mixing of jet with the ambient fluid only in the characteristic decay zone but not in the core and fully developed zones.

2. Elliptical and rectangular orifice show different mixing characteristics.

3. The core region is same for both shapes.

4. The level of mixing retardation was found to be maximum when wall was placed parallel to the major axis plane of the jet.

5. The effect of wall on jet mixing was intense for rectangular jet compared to elliptic jet.

\section{Future Enhancement}

1. Characteristic region should be the main focus when studying subsonic jets so any further work to be done should be studied in this region.

2. More shapes can be tried out

3. More applications should be found out where less mixing is required.
4. This project can be repeated for supersonic case.

5. The literature behind the graphs can be researched in detail.

\section{References}

[1] Glauert, M. B., "The wall jet," Journal of Fluid Mechanics, vol. 1, pp. 625-643, 1956

[2] Yoon S.H., Kim K.C., Kim D.S., Chung M.K., " Effect of Surface Roughness on a Turbulent Wall-attaching Offset Jet". Experiments in Fluids, Vol. 19, 1995, pp. 38-42.

[3] Benjamin Scott smith, "Wall jet boundary layer flows over smooth and rough surfaces", Ph.D. Thesis, Virginia Polytechnic, 2008

[4] Sakakibar, Y., Endo, M. and Iwamoto, J., "Effect of Wall Jet on Oscillation Mode of Impinging Jet", 10th FLUCOME, Moscow, Russia, 2009

[5] Teles, J. C. M. G., "The turbulent axisymmetric wall jet," M.Sc. Thesis, Imperial College of Science and Technology Mechanical Engineering Department, London 